\title{
MODELOS DA QUÍMICA QUÂNTICA NO ESPAÇO DE MOMENTO: DIFERENTES REPRESENTAÇÕES DE UM MESMO SISTEMA
}

\author{
Willian Hermoso e Fernando R. Ornellas* \\ Departamento de Química Fundamental, Instituto de Química, Universidade de São Paulo, CP 26077, 05513-970 São Paulo - SP, \\ Brasil
}

Recebido em 10/12/08; aceito em 18/5/09; publicado na web em 1/10/09

\begin{abstract}
QUANTUM CHEMISTRY MODELS IN THE MOMENTUM SPACE: DIFFERENT REPRESENTATIONS OF THE SAME SYSTEM. The conventional approach to simple quantum chemistry models is contrasted with that known as momentum representation, where the wavefunctions are momentum dependent. Since the physical interactions are the same, state energies should not change, and whence the energy differences correlating with the real world as spectral lines or bands. We emphasize that one representation is not more fundamental than the other, and the choice is a matter of mathematical convenience. As spatial localization is rooted in our brains, to think in terms of the momentum present us a great mental challenge that can lead to complementary perspectives of a model.
\end{abstract}

Keywords: momentum representation; coordinate representation; quantum chemistry models.

\section{INTRODUÇÃO}

O ensino de Química Quântica é hoje um componente fundamental na formação dos estudantes de Química, pois a compreensão de fenômenos químicos e físicos sob uma óptica microscópica requer necessariamente uma abordagem quântica. Tradicionalmente, o aluno tem seus primeiros contatos com conceitos de Química Quântica ao estudar sistemas fisicamente simples que permitem ilustrar uma maneira de pensar distinta daquela da física clássica. Obviamente, nessa fase, e até mesmo em cursos ou textos mais avançados, não é salientado que o desenvolvimento teórico a que está sendo introduzido corresponde ao que se convencionou chamar espaço das coordenadas ou representação da posição. Sem nos preocuparmos com rigores de formalismo, podemos dizer que, nessa representação, a função de onda associada à descrição do estado de um sistema é função das posições das partículas que o constituem. Isso, naturalmente, induz o aluno a adquirir uma falsa impressão que a descrição dos sistemas na Química Quântica está sempre baseada na posição de cada uma das partículas, não havendo outra possibilidade de caracterização. Neste artigo, mostramos que a estrutura matemática da Mecânica Quântica nos permite apresentar a Equação de Schrödinger numa outra forma praticamente desconhecida do químico, em que a função de estado não é mais função das coordenadas das partículas, mas sim de seus momentos lineares. Essa apresentação alternativa é conhecida como espaço ou representação de momento. Como as interações físicas que caracterizam o sistema permanecem inalteradas, as energias (autovalores da Equação de Schrödinger) próprias de cada estado têm que ser invariantes à forma de representação.

Aqui, em particular, vamos examinar os modelos do oscilador harmônico, do átomo de hidrogênio e o de uma partícula numa caixa unidimensional e mostrar que não precisamos ter nosso raciocínio enraizado numa descrição em termos das posições das partículas que compõem o sistema para caracterizar energeticamente seu estado. Essa perspectiva obviamente leva a uma visão não-usual que é importante para ilustrar ao aluno a distinção entre modelo e realidade e a conveniência de se trabalhar numa ou noutra das representações. Apesar de nesse contexto a transição de uma para outra representação ser novidade para o aluno, o conceito matemático de representação pode ser encontrado na Termodinâmica ao tratar os conceitos de

*e-mail: frornell@usp.br entropia, energia de Gibbs e energia de Helmholtz, por exemplo. Todas são funções termodinâmicas que caracterizam um sistema e nos permitem definir condições de equilíbrio e espontaneidade, mas a escolha de uma ou outra é uma questão de conveniência prática ou teórica que, matematicamente, significa a escolha de uma ou outra representação e que, nesse caso, é definida através de uma operação matemática conhecida como transformação de Legendre. ${ }^{1}$

\section{ASPECTOS TEÓRICOS}

Pelo fato de não ter familiaridade com equações diferenciais, a Equação de Schrödinger independente do tempo, como apresentada abaixo (em uma dimensão por questão de simplicidade), tem um impacto assustador no estudante de química.

$-\frac{\hbar^{2}}{2 m} \frac{\partial^{2} \Psi(x)}{\partial x^{2}}+V(x) \Psi(x)=E \Psi(x)$

Numa sequiência lógica, o significado físico de cada um de seus termos é apresentado e uma concepção nova de representação de variáveis físicas em termos de operadores matemáticos (diferenciais e multiplicativos) é introduzida. Nesse contexto, ao aluno é ensinado que, em uma dimensão, a posição de uma partícula será associada ao operador multiplicativo $x$, e seu momento linear ao operador diferencial $-i \hbar \partial / \partial x{ }^{2}$ Como classicamente a energia cinética de um sistema é igual a $p^{2} / 2 m$, mostra-se ao aluno que essa associação é coerente com o termo do operador de energia cinética $\left(-\hbar^{2} / 2 m\right) \partial^{2} / \partial x^{2}$ na Equação 1. Muitas vezes, nessa fase mostra-se, também, que esses operadores satisfazem uma relação de comutação fundamental na mecânica quântica, expressa na Equação 2, cuja interpretação nos diz que é impossível conhecer simultaneamente, com precisão arbitrária, a posição e o momento linear de uma partícula.,34

$\left[-i \hbar \frac{\partial}{\partial x}, x\right]=-i \hbar$

Uma vez apresentada, a seguir passa-se à aplicação dessa equação a alguns sistemas simples, enfatizando que: (1) a quantização da energia surge de forma natural; (2) a função de onda $\Psi(x)$ não tem uma interpretação física associada a ela; e, (3) que $\Psi(x) * \Psi(x)$ representa uma distribuição de probabilidade, sendo $\Psi(x)^{*} \Psi(x) d x$ a 
probabilidade de encontrar a partícula entre $x$ e $x+d x$. Neste ponto, é oportuno o professor enfatizar que, em contraste com a mecânica clássica, a equação de onda não descreve a dinâmica da partícula (como ela se movimenta, ou mais simplesmente, "o que ela faz"). Com essas soluções podemos somente fazer previsões probabilísticas sobre a ocorrência de um dado evento e determinar médias das variáveis associadas às quantidades físicas de interesse. É interessante também enfatizar que a conexão da teoria com o mundo atômico e molecular se faz através de diferenças de energia entre os estados de um sistema; essas diferenças de energia podem ser associadas às linhas ou bandas nos diferentes tipos de espectros, ou determinadas como entalpias no caso de reações.

Fazemos notar, entretanto, que um aluno com um pouco mais de curiosidade matemática poderia ter apontado ao professor que ele verificou que o par de operadores ( $p$ e $i \hbar \partial / \partial p)$ também satisfaz a relação (2) e que fisicamente esses operadores poderiam ser associados ao momento linear e à posição da partícula, o que, obviamente, levaria a uma Equação de Schrödinger distinta da Equação 1. De fato, a Equação 3 é uma forma equivalente à Equação 1 em que a função de onda e os operadores de energia cinética e potencial são agora expressos em função do momento linear da partícula. ${ }^{5}$

$$
\frac{p^{2}}{2 m} \Phi(p)+\int U\left(p-p^{\prime}\right) \Phi(p) d p^{\prime}=E \Phi(p)
$$

Aqui, o termo correspondente à energia cinética tem semelhança com a expressão clássica, mas o termo associado à energia potencial assume agora a forma de um operador integral. Na Equação 3, $U(p$ $-p^{\prime}$ ) é conhecido como kernel da integral e é dado por

$U(p)=\int_{-\infty}^{\infty} V(x) e^{-i p x / \hbar} d x$

em que $V(x)$ é a energia potencial da Equação 1 .

Essa perspectiva distinta de descrição do estado de um sistema em termos de funções de onda dependentes do momento da partícula pode ser obtida através da resolução da Equação integral 3 ou, alternativamente, através da transformada de Fourier da correspondente solução no espaço de coordenadas ou da posição, definida pela relação (5), dada em uma dimensão por simplicidade. ${ }^{6}$

$\Phi(p)=\frac{1}{\sqrt{2 \pi \hbar}} \int \Psi(x) e^{-i p x / \hbar} d x$

\section{ESPAÇO DO MOMENTO: UM ESTUDO DE CASOS}

Nesta seção, vamos ilustrar uma forma alternativa, mas equivalente, de descrição de três modelos simples amplamente discutidos nos cursos de Química Quântica, mais especificamente, o oscilador harmônico, o átomo de hidrogênio e uma partícula confinada numa caixa unidimensional com paredes infinitas. Procuraremos nos limitar às equações mais essenciais; detalhes matemáticos sobre a obtenção das equações e as transformações realizadas podem ser encontrados na ref. 7.

\section{Oscilador harmônico}

A Equação de Schrödinger para um oscilador harmônico, mostrada abaixo, é amplamente discutida e sua solução apresentada em detalhes nos cursos iniciais de Química Quântica.

$-\frac{\hbar^{2}}{2 \mu} \frac{\partial^{2} \Psi(x)}{\partial x^{2}}+\frac{k x^{2}}{2} \Psi(x)=E \Psi(x)$
A resolução dessa equação leva naturalmente à quantização da energia do oscilador, expressa como $E(v)=(v+1 / 2) \hbar \omega$, em que $\omega$ é a frequência angular, e $v=0,1,2,3, \ldots$ é o número quântico vibracional. Como bem conhecido, sua solução geral tem a forma

$\Psi_{v}(x)=N_{v} H_{v}(x) \exp \left\{-\alpha x^{2} / 2\right\}$

em que $\alpha$ é igual a $\left(\frac{\mu k}{\hbar^{2}}\right)^{1 / 2}, N_{v}$ é a constante de normalização, dada pela relação $N_{v}=\left(2^{v} v ! \sqrt{\pi / \alpha}\right)^{1 / 2}$ e $H_{v}(x)$ é o polinômio de Hermite de grau $v$

Sabendo que na representação do momento a posição da partícula é associada ao operador diferencial $i \hbar \partial / \partial p$ e o momento linear ao operador multiplicativo $p$, pode-se mostrar que a Equação 3 para o caso específico do oscilador harmônico toma a seguinte forma: ${ }^{7}$

$$
\frac{p^{2}}{2 \mu} \Phi(p)-\frac{k \hbar^{2}}{2} \frac{\partial^{2} \Phi(p)}{\partial p^{2}}=E \Phi(p)
$$

em que $\mu$ é a massa reduzida da sistema, $k$ é a constante de força do oscilador e $E$ sua energia.

Neste caso, em particular, chamamos a atenção primeiro para a forma matemática das duas Equações 6 e 8. Deve-se notar que o termo correspondente à energia cinética na representação de coordenadas, expresso na forma de um operador diferencial, é agora associado a um operador multiplicativo; no caso do operador multiplicativo associado à energia potencial $\left(1 / 2 k x^{2}\right)$, sua representação no espaço do momento toma a forma de um operador diferencial. ${ }^{7}$ Do ponto de vista matemático, essas duas equações são idênticas e o mesmo procedimento apresentado nos livros-textos para a resolução da equação no espaço de coordenadas se aplica à equação no espaço do momento, levando à mesma expressão para a energia escrita acima e às soluções

$\Phi_{v}(p)=N_{v} e^{-\alpha^{\prime} p / 2} H_{v}\left(\alpha^{\prime} p\right)$

em que $\alpha^{\prime}$ é igual a $\left(\frac{1}{\hbar^{2} \alpha}\right)$. Ressaltamos, novamente, que a energia associada a certo estado $v$ independe de qual representação se esteja trabalhando. Restringindo-nos, por simplicidade, ao estado fundamental $\left(\Psi_{0}\right)$, temos

$\Phi_{0}(p)=\left(\alpha^{\prime} / \pi\right)^{-1 / 4} e^{-\alpha^{\prime} p^{2} / 2}$

que tem a mesma forma analítica da solução no espaço de coordenadas mostrada abaixo.

$\Psi_{0}(x)=(\alpha / \pi)^{-1 / 4} e^{-\alpha x^{2} / 2}$

Alternativamente, essa mesma solução poderia ser obtida através da transformada de Fourier definida pela Equação 5, ou seja,

$\Phi_{0}(p)=\frac{1}{\sqrt{2 \pi \hbar}} \int_{-\infty}^{\infty} \Psi_{0}(x) e^{-i p x / \hbar} d x$

Aqui se pode salientar a propriedade da transformada de Fourier de uma função do tipo gaussiana gerar outra função também do tipo gaussiana. ${ }^{6}$

Na Figura 1 ilustramos as distribuições de probabilidades no espaço do momento para vários estados do oscilador harmônico, resultado esse nunca apresentado nos livros-textos. Nos gráficos deve-se notar que apesar da energia de cada estado ser bem conhecida, o momento da partícula não é bem definido, o que é bem contrastante com a previsão determinista da mecânica clássica. Sendo uma distribuição, 
a simetria embutida nesses gráficos mostra claramente que o valor médio do momento em qualquer estado será sempre zero, resultado esse que é normalmente proposto como um exercício a ser provado no espaço de coordenadas. Nota-se também que, conforme o número quântico vibracional aumenta, o sistema tende a se aproximar de um comportamento clássico, obedecendo assim ao princípio da correspondência. ${ }^{8}$ Da forma analítica semelhante das funções de onda nas duas representações podemos também inferir que o cálculo de probabilidades de transições levará à mesma regra de seleção $(\Delta v= \pm 1){ }^{3}$

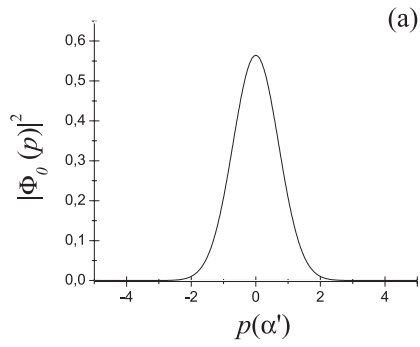

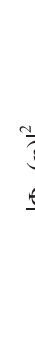
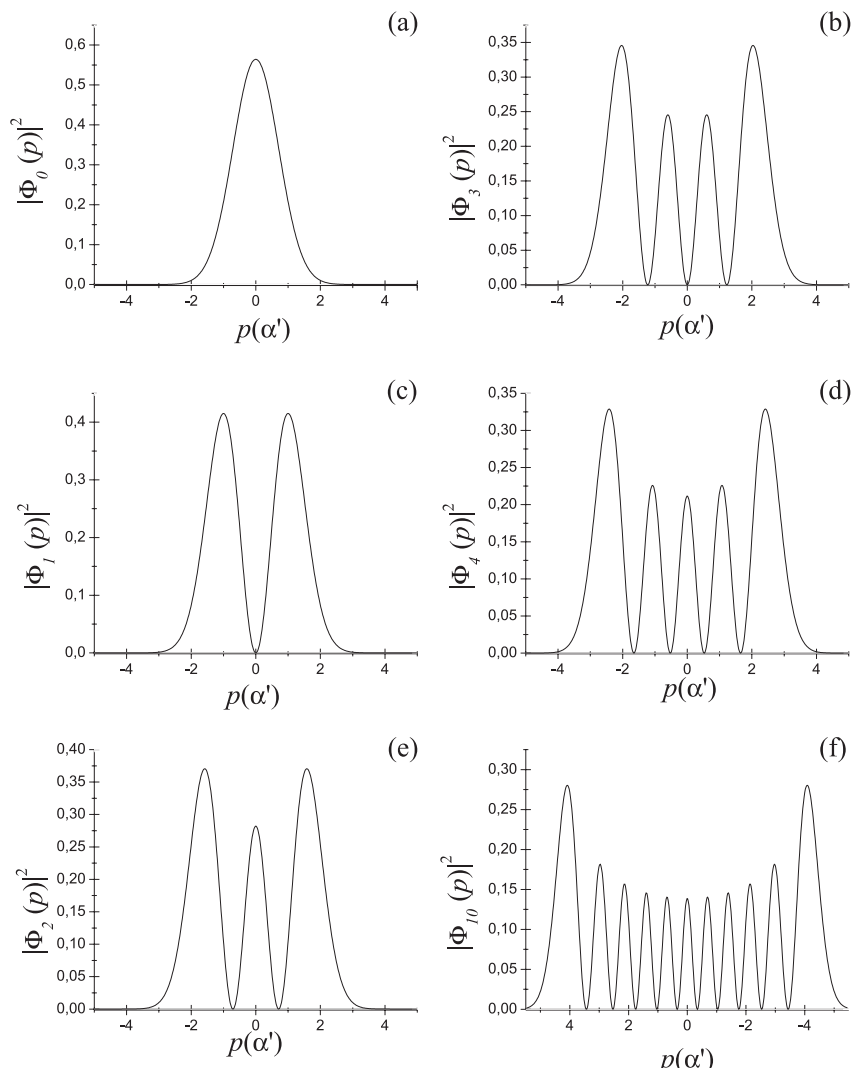

(e)

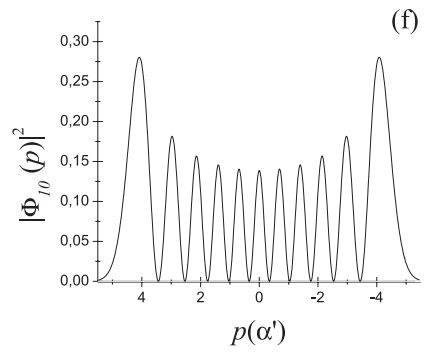

Figura 1. Distribuições de probabilidades do momento do oscilador harmônico para os estados $v=0,1,2,3,4$ e 10

Neste modelo, a semelhança das equações nas duas representações torna irrelevante a escolha de uma abordagem ou outra. Entretanto, poderíamos imaginar que se por algum fator operante na evolução da mecânica quântica (ou de nosso cérebro) somente a equação no espaço de momento tivesse sido proposta, o pensar em termos do deslocamento do oscilador (p.ex., das posições dos átomos num sistema diatômico) certamente não estaria tão enraizado em nosso subconsciente e não acharíamos estranho raciocinar em termos do momento, como o achamos agora. Como tudo o que é previsto numa representação pode ser também previsto na outra, nenhuma delas é mais fundamental ou mais relevante que a outra. A escolha entre uma e outra é uma questão de conveniência ou de preferência pessoal.

\section{Átomo de hidrogênio}

O modelo do átomo de hidrogênio é o caso protótipo para a compreensão inicial de espectros e de sistemas atômicos mais complexos. Ao ser exposto primeiro ao modelo de Bohr, o estudante é confrontado ao mesmo tempo com o sucesso momentâneo e com as limitações de tal modelo. ${ }^{9}$ Mais adiante, ao ser introduzido à Equação de Schrödinger, vivencia outro choque, o da generalidade de aplicação da equação simultaneamente à perda de uma visualização clássica. Um novo paradigma surge. Uma nova forma de pensar é então construída. Apesar da impossibilidade de análise clássica imposta pela Mecânica Quântica, o resultado relevante que conecta a teoria com o mundo real, o espectro atômico, é corretamente previsto.

As Equações 13 e 14 contrastam a forma da Equação de Schrödinger nas representações de posição e de momento para o átomo de hidrogênio:

$$
\begin{aligned}
& -\frac{\hbar^{2}}{2 \mu} \nabla^{2} \Psi(\mathbf{r})+\frac{e^{2}}{4 \pi \varepsilon_{0}} \Psi(\mathbf{r})=E \Psi(\mathbf{r}) \\
& \left(-k^{2}+a^{2}\right) \Phi(\mathbf{p})=\frac{\beta^{2}}{h \pi} \int \frac{\Phi\left(\mathbf{p}^{\prime}\right)}{|\vec{p}-\vec{p}|^{2}} d^{3} p^{\prime}
\end{aligned}
$$

A Equação 13 é analisada em detalhes nos cursos de Química Quântica; ;,4,8-10 já a Equação 14 foi resolvida por Fock, ${ }^{11}$ logo no começo da nova teoria quântica. Funções de onda no espaço de momento, obtidas via transformada de Fourier, foram ainda estudadas por Podolsky e Pauling ${ }^{12}$ também nos primórdios da mecânica quântica. A solução matemática da Equação 14 é extremamente complexa. ${ }^{7}$ Daí, talvez, sua não-popularidade nos livros-textos de Química Quântica. Entretanto, o ponto essencial que queremos destacar é que, sendo a função de onda no espaço de momento dependente do momento do elétron, $\Phi(p)^{*} \Phi(p)$ vai nos fornecer uma distribuição de probabilidade do momento que contrasta com nosso pensar inconsciente de tentar associar a partícula a algum ponto no espaço, como ocorre na outra representação. Para podermos examinar um pouco mais esse aspecto, vamos analisar as soluções para o átomo de hidrogênio nos dois espaços, restringindo-nos, por simplicidade, ao estado fundamental. As Equações 15 e 16 são duas funções com variáveis bem distintas, mas que corretamente descrevem o estado fundamental do átomo de hidrogênio, ou seja, ambas predizem o valor $-0,5$ u.a. para a energia do átomo no estado fundamental.?

$$
\begin{aligned}
& \Psi_{1 s}(\mathbf{r})=\frac{1}{\sqrt{\pi a_{0}}} e^{-r / a_{0}} \\
& \Phi_{1 s}(\mathbf{p})=\sqrt{\frac{8 \hbar^{5} a_{0}^{3}}{\pi}}\left[\frac{1}{\left(\hbar^{2}+p^{2} a_{0}^{2}\right)^{2}}\right]
\end{aligned}
$$

Como o pensar quântico é probabilístico, na Figura 2 mostramos as distribuições radiais correspondentes a essas duas funções de estado.
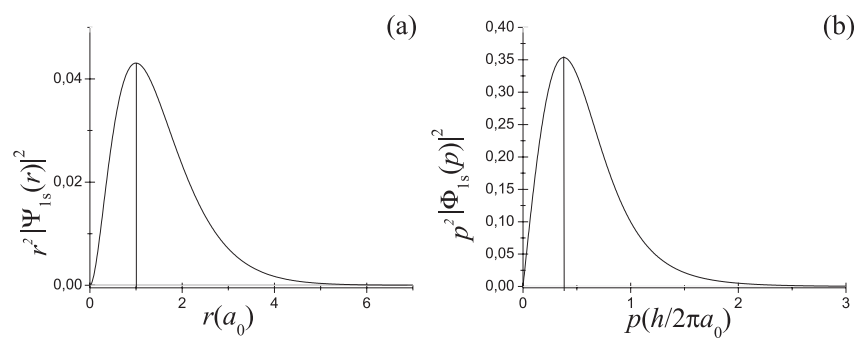

Figura 2. Distribuições radiais de probabilidades do átomo de hidrogênio para o estado fundamental

Nos cursos usuais, o máximo na distribuição radial no espaço de coordenadas é interpretado como indicativo da posição mais provável de encontrar o elétron no estado fundamental. Acidentalmente (ou reflexo de algo mais profundo), a distância correspondente a esse máximo é exatamente igual ao raio calculado no modelo de Bohr. Por coerência lógica na estrutura da Mecânica Quântica, a mesma forma interpretativa vale para a distribuição no espaço de momento, 
ou seja, o máximo na distribuição do momento está associado ao valor (absoluto) mais provável do momento. Usando essa distribuição para calcular o valor médio de $p^{2} / 2 m$, obtemos a energia cinética média, que pelo teorema do virial é igual ao negativo da energia total do sistema. Note que ambas as descrições são equivalentes, nenhuma é mais fundamental que a outra. Ambas fornecem as mesmas energias para o átomo de hidrogênio e, dessa forma, fazem a conexão do modelo com o mundo real, ou seja, o espectro do átomo de hidrogênio. Pensar em termos do momento do elétron é algo não-usual e até certo modo difícil de ser concebido. Como a localização no espaço é algo que trazemos inconscientemente, pensar em termos da distribuição da posição nos parece mais familiar, mas é importante enfatizar que a Mecânica Quântica não nos diz o que o elétron está fazendo no átomo, nem como ele se movimenta. Quando um elétron é excitado do estado $1 s$ para o estado $2 p$, por exemplo, ela não nos diz como isso ocorre, mas somente a probabilidade de sua ocorrência e nas duas representações nós somos capazes de fazer a previsão correta. Como já enfatizado, matematicamente é mais fácil trabalhar na representação das coordenadas, mas deve ficar claro que ela, e toda a interpretação física resultante, não são mais fundamentais que as correspondentes no espaço de momento.

\section{Partícula numa caixa unidimensional}

A descrição quântica de uma partícula confinada numa caixa unidimensional de largura $L$, em que o potencial é igual a zero entre os extremos $-L / 2$ e $L / 2$ e infinito fora desse intervalo, estando, portanto, livre para se movimentar em seu interior, é o primeiro modelo examinado em detalhes num curso de Química Quântica e ilustra de forma simples a quantização da energia em sistemas confinados. As Equações de Schrödinger correspondentes às duas representações são mostradas abaixo:

$$
\begin{aligned}
& -\frac{\hbar^{2}}{2 m} \frac{d^{2} \Psi(x)}{d x^{2}}=E \Psi(x) \\
& \frac{p^{2}}{2 m} \Phi(p)=E \Phi(p)
\end{aligned}
$$

A Equação 17 é uma das primeiras introduzidas ao aluno e, mesmo que não saiba nada de equações diferenciais, ela é muito fácil de ser resolvida; basta que ele faça a pergunta: que tipo de função $\Psi(x)$, que derivada duas vezes, resulte em $-2 m E / \hbar^{2} \Psi(x)$ ? Sua solução é usualmente apresentada na forma $\Psi(x)=\mathrm{A}$ sen $k x+\mathrm{B} \cos k x$, em que $k^{2}=2 m E / \hbar^{2}$. Como conhecido, a imposição das condições de contorno, $\Psi(-L / 2)=\Psi(L / 2)=0$, introduz o par de restrições $(\mathrm{B}=$ 0 e $k L / 2=n \pi \operatorname{com} n=2,4,6, \ldots)$ e $(\mathrm{A}=0$ e $k L / 2=n \pi \operatorname{com} n=1,3$, $5, \ldots)$, que leva à quantização da energia $\left(E_{n}=n^{2} h^{2} /\left(8 m L^{2}\right)\right) \cdot{ }^{3,4,8-10} \mathrm{No}$ espaço de momento, entretanto, apesar da equação ter uma aparência mais simples, sua solução não é de imediato percebida pelo estudante sem o conhecimento de propriedades da função delta de Dirac. ${ }^{6}$ Se o intervalo em que o potencial é zero se estendesse infinitamente, nosso problema se transformaria no de uma partícula livre e a solução da Equação 18 seria igual a

$\Phi(p)=B \delta\left[\left(p+p_{0}\right)+\left(p-p_{0}\right)\right]$

que, fisicamente, representa uma onda plana (partícula) com igual probabilidade de estar se movendo nas direções $\pm x$. No interior da caixa (energia potencial igual à zero), a forma funcional da solução deve ser semelhante à da solução 19 , mas devidamente modificada para refletir as condições de contorno do problema. Entretanto, a imposição dessas condições está longe de ser trivial ${ }^{13}$ e não será discutida aqui. No entanto, para que possamos analisar a solução no espaço de momento, vamos expressá-la como a transformada de Fourier da solução no espaço de coordenadas.

$$
\Phi_{n}(p)=\frac{1}{\sqrt{2 \pi \hbar}} \int_{-\infty}^{\infty} \Psi_{n}(x) e^{-i p x / \hbar} d x
$$

Devido à simetria da caixa, as soluções no espaço de coordenadas são expressas como dois conjuntos de funções (pares e ímpares); de forma semelhante, no espaço de momento podemos também escrever

$$
\begin{gathered}
\Phi_{n}(p)=\frac{(-1)^{\frac{n-1}{2}}}{\pi} \sqrt{\frac{2 h}{a}} \frac{p_{n} \cos (p a / 2 \hbar)}{p_{n}{ }^{2}-p^{2}}, \mathrm{n}=1,3,5 \ldots \\
\Phi_{n}(p)=\frac{i(-1)^{n / 2}}{\pi} \sqrt{\frac{2 h}{a}} \frac{p_{n} \operatorname{sen}(p a / 2 \hbar)}{p_{n}{ }^{2}-p^{2}}, \mathrm{n}=2,4,6 \ldots
\end{gathered}
$$

Embora não pareça claro numa primeira inspeção, a descrição do estado de uma partícula nessa caixa, usando as funções 21 e 22, é totalmente equivalente à feita pelas funções de onda expressas em termos da posição da partícula. Novamente, a grande dificuldade é que nosso cérebro não foi educado para pensar no espaço do momento. Apesar dessa dificuldade, vamos procurar ilustrar como essas funções podem nos ajudar a apontar um erro recorrente nos livros-textos. ${ }^{14-18}$

Ao analisar as soluções pares $(\cos k x)$, por exemplo, e fazendo uso da relação

$\cos \alpha=\frac{e^{i \alpha}+e^{-i \alpha}}{2}$

interpreta-se essa solução como uma superposição de duas ondas planas $\left(\mathrm{e}^{i k x}+\mathrm{e}^{-i k x}\right)$ movendo-se em direções opostas, em que o valor de $k$ está fixo pela energia associada ao estado através da relação $k^{2}=$ $2 m E / \hbar^{2}$. Essa interpretação é incorreta ${ }^{14-18} \mathrm{e}$, infelizmente, encontrada em vários livros-textos. Para verificar por que é incorreta basta olhar as distribuições de momento para vários estados mostradas na Figura 3. Nesses gráficos, nota-se claramente que todas elas são indicativas de que o momento não tem um valor fixo, mas varia dentro de um dado intervalo. À medida que o sistema se aproxima do limite clássico (n $\rightarrow \infty$ ou $L \rightarrow \infty$ ), essa distribuição se estreita e se reduz a funções- $\delta$ centradas em $\pm k .{ }^{16}$
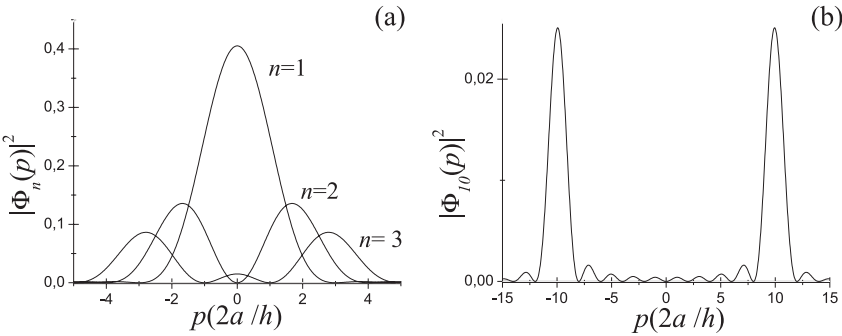

Figura 3. Distribuições de probabilidades do momento para uma partícula numa caixa unidimensional para os estados $n=1,2,3$ e 10

De passagem, salientamos também que o modelo da partícula numa caixa unidimensional tem ainda problemas adicionais como a descontinuidade da derivada primeira e da segunda (em relação à $x$ ) nos pontos extremos que, normalmente, não são apresentados nos livros-textos usuais. Sua discussão também envolve uma abordagem matemática mais avançada, que poderá ser estudada nas referências originais pelo leitor com um maior embasamento teórico. Apesar de não rigoroso, o procedimento usual para a obtenção da energia não é afetado. ${ }^{17,18}$ 


\section{Conexão com o mundo real}

Já ressaltamos nas seções anteriores que diferenças de energias, obtidas pela solução da Equação de Schrödinger, se correlacionam com o mundo real através das linhas e bandas espectrais. É interessante ainda destacar que distribuições de momento podem ser determinadas experimentalmente. Uma dessas técnicas baseia-se no chamado efeito ou espalhamento Compton, ${ }^{19,20}$ em que se observa uma diminuição na frequência da radiação (raios X) espalhada, em relação à incidente, após sua colisão com elétrons num determinado alvo. Na realidade, observa-se um alargamento da linha da radiação espalhada, resultando, assim, numa banda de espalhamento. $\mathrm{Na}$ literatura, essa banda é denominada perfil de Compton e é expressa pela relação abaixo derivada com o auxílio da Mecânica Quântica:

$J(q)=\frac{1}{2} \int_{|q|}^{\infty} \frac{I(p)}{p} d p$

em que $q$ é um comprimento de onda efetivo e $I(p)$ é a distribuição radial de momento, que pode ser calculada conhecendo-se a função de onda no espaço de momento. A outra técnica é conhecida como espectroscopia de momento de elétron, ou espectroscopia de coincidência eletrônica, simbolizada por (e, 2e). ${ }^{21-23}$ Aqui, elétrons são direcionados a um alvo de onde ejetam outros elétrons e se espalham. Com um arranjo experimental apropriado, o elétron espalhado e o elétron ejetado são detectados simultaneamente. Usando leis de conservação de momento e de energia, é possível determinar a energia e o momento do elétron ejetado antes da colisão. Como a taxa de elétrons espalhados numa dada direção é expressa pela seção de choque diferencial $(d \sigma / d \Omega)$, pode-se provar teoricamente que essa seção de choque está relacionada à função de onda no espaço de momento pela relação

$d \sigma / d \Omega=\mathrm{C}|\Phi(p)|^{2}$

que ressalta, mais uma vez, o papel central dessa função na análise de dados experimentais nesse tipo de experimento.

\section{CONCLUSÕES}

Neste trabalho, procuramos mostrar uma alternativa distinta de apresentação e discussão de alguns modelos simples estudados em cursos introdutórios de Química Quântica. Pensar em termos das posições das partículas (ou de elétrons em átomos e moléculas) não é uma condição necessária para se descrever sistemas quânticos. O uso de uma ou outra das duas representações aqui discutidas não implica que uma seja mais fundamental ou mais importante que a outra, mas sim que o ferramental matemático é mais simples de ser tratado. Pensar em termos de funções de onda que dependam do(s) momento(s) da(s) partículas é obviamente um grande exercício mental, principalmente quando entramos no universo químico, visto que temos enraizado em nosso cérebro a localização espacial. Sair do tratamento usual e analisar uma ligação química no espaço de momento se constitui num problema bastante desafiador, que pode trazer uma perspectiva complementar à nossa abordagem tradicional desse universo, mas isso é um problema que voltaremos a abordar num próximo trabalho.

\section{AGRADECIMENTOS}

W. Hermoso agradece ao CNPq pela concessão de uma bolsa de mestrado e F. R. Ornellas é grato ao CNPq e à FAPESP pelo constante apoio acadêmico. Aos membros do grupo pelas discussões animadas e enriquecedoras.

\section{REFERÊNCIAS}

1. McQuarrie, D. A.; Statistical Mechanics, $1^{\text {st }}$ ed., Harper \& Row: New York, 1976.

2. Ornellas, F. R.; Quim. Nova 1990, 13, 211.

3. Pilar, F. L.; Elementary Quantum Chemistry, $2^{\text {nd }}$ ed., Dover: New York, 2001.

4. Levine, I. N.; Quantum Chemistry, $4^{\text {th }}$ ed., Prentice Hall: New Jersey, 1991.

5. Guillaumín-España, E.; Salas-Brito A. L.; Martínez y Romero, R. P.; Núñez-Yépez, H. N.; Rev. Mex. Fís. 2001, 47, 98.

6. Arfken, G. B.; Weber, H. J.; Mathematical Methods for Physicists, $6^{\text {th }}$ ed., Elsevier: San Diego, 2005.

7. Hermoso, W.; Dissertação de Mestrado, Universidade de São Paulo, Brasil, 2008.

8. Schiff, L. I.; Quantum Mechanics, $3^{\text {rd }}$ ed., McGraw-Hill: New York, 1968.

9. Pauling, L.; Wilson, E. B. Jr.; Introduction to Quantum Mechanics: with applications to chemistry, Reprint ed., Dover: New York, 1985.

10. McQuarrie, D. A.; Quantum Chemistry, $1^{\text {st }}$ ed., University Science Books: Mill Valley, 1983.

11. Fock, V.; Z. Physik 1935, 98, 145

12. Podolsky, B.; Pauling, L.; Phys. Rev. 1929, 34, 109.

13. Cummings, F. E.; Am. J. Phys. 1977, 45, 158.

14. Markley, F. L.; Am. J. Phys. 1972, 40, 1545.

15. Johannin-Gilles, A.; Abjean, R.; Guern, Y.; Leriche M.; Am. J. Phys. 1974, 42, 702 .

16. Liang, Y. Q.; Zhang, H.; Dardenne, Y. X.; J. Chem. Educ. 1995, 72, 148.

17. Muha, G. M.; J. Chem. Educ. 1984, 61, 414.

18. Seki, R.; Am. J. Phys. 1971, 39, 929.

19. Cooper, M.; Adv. Phys. 1971, 20, 453.

20. Epstein, I. R.; Acc. Chem. Res. 1973, 6, 145.

21. McCarthy, I. E.; Weigold, E.; Contemp. Phys. 1983, 24, 163.

22. Lohmann, B.; Weigold, E.; Phys. Lett. A 1981, 86, 139.

23. Weigold, E.; Hood, S. T.; McCarthy, L. E.; Teubner, P. J. O. Phys. Lett. A 1973, 44, 531. 